\title{
Behavior Based Detection of Unfavorable Resources
}

\author{
Krzysztof Cetnarowicz ${ }^{1}$ and Gabriel Rojek ${ }^{2}$ \\ 1 Institute of Computer Science, \\ AGH University of Science and Technology, \\ Al. Mickiewicza, 30 30-059 Kraków, Poland \\ cetnar@agh.edu.pl \\ 2 Department of Computer Science in Industry, \\ AGH University of Science and Technology, \\ Al. Mickiewicza 30, 30-059 Kraków, Poland \\ rojek@agh. edu.pl
}

\begin{abstract}
This article considers a problem of security in a computer systems in context of some mechanisms which act in societies and which mechanisms are seen as being useful to assure security in a computer system. A new approach to the security problem is discussed, which refers to identifying computer resource (e.g. agent, program) on the basis of this resource behaviour (actions which this resource undertakes). Mechanisms are presented, which may enable to recognize and dismiss resources undesirable or harmful in the computer system on the basis of behavior observation. Proposed mechanisms were tested in simulations of the computer system under DoS attack. The results of tests are presented and discussed.
\end{abstract}

\section{Introduction}

Present computer systems have become more and more complex and related to each other. Large nets, as well as almost all devices connected to the Internet, are open systems. It is impossible to have full knowledge about topology or current state of such systems even for the owner, or an administrator of a computer system [5]. Agent technology makes possible the full flow of resources among open computer systems. Autonomic agents can yet freely migrate in the net without the knowledge of the owner or an administrator. Agents can also execute their tasks without anybody's knowledge. These tasks could be useful or destructive for the system on which an agent operate. An agent which migrate in an open system could be equally desirable or undesirable in a computer system. This ambiguousness causes problems with the use of artificial immune systems which makes possible to distinguish two classes of resources: self or nonself 3467) 8. In the case of migrating agent in open system, the origin of supply (self / nonself) does not play an essential part in the process of assuring security [2]. 


\section{Social Approach to Security Problem}

In the introduction mentioned facts and trends lead to the formulation of a new foundation of solution of computer's security problem. Attacks have generally decentralized nature and harmful resources (which cause these attacks) should be seen as in some way autonomous agents. In order to oppose against such threats, the computer system should be seen as a multiagent system, which consists of:

- "good" (desirable) resources (e.g. agents, programs),

- "bad" (undesirable) resources,

as it was proposed in [2].

Taking into consideration the above-mentioned problems with security systems, which are centralized and operate on fragments of code (look for known signatures), a way is proposed, how the division "good" / "bad" should be made. It could be stated, that:

- decentralized security system could be realized in the form of functions, mechanisms or algorithms that are build in as big amount of components of protected system as possible;

- division "good" / "bad" could be based on observation and evaluation of behavior of components of the protected system.

Authors formulating above mentioned paradigms where inspired by some of ethically-social mechanisms that act in human societies. That mechanisms prevent from misuses in societies in the way to enable secure functioning of an individual in the environment of others individuals. Some of individuals in society could be prejudicial for others individuals, what is main analogy to the society of agents, programs and other resources in a computer system.

An individual in a society seems trustworthy if behavior of this individual could be observed by other individuals in a society and this behavior is evaluated by majority as good and secure 910. The decision about trustworthy of an individual are in society made in the decentralized way - all individuals in a society make own decisions which form one decision of the society. The decisions undertaken by individuals in the societies are made on the ground of observation and evaluation of behavior (and / or intentions) of the individual which decisions concerns.

\subsection{Decentralization of Security System}

Decentralization paradigm could be realized in multiagent algorithms by means of equipping all agents (agents that exist in the protected system) with some additional goals, tasks and mechanisms. Those goals, tasks and mechanism are named a division profile and should be designed to assure security for agents and the computer system, which those agents assemble. So the agents will execute tasks they have been created for and simultaneously will execute tasks connected with security of all agents in the system and / or the computer system. The name "division profile" is inspired by M-agent architecture which could be used to describe an agent (M-agent architecture was introduced among others in [1]). 


\subsection{Observation and Evaluation of Behavior}

Undertaking actions by an acting agent should be seen as objects. Those objects create a sequence which could be registered by agents observing that acting agent. Registered objects-actions could be processed in order to qualify whether it is a "good" or "bad" acting agent. It should be mentioned, that the quoted notions of "good" and "bad" do not have absolute meaning. "Good" resource is a desirable resource for a definite computer system in which evaluation takes place. "Bad" resource is an undesirable resource for a given system, although it can happen that it would be greatly desirable in a different computer system.

\section{Division Profile}

Division profile is a class of agent activity whose goal is to observe others agents in society and possible other elements of the environment. Those observations should be made in order to distinguish individuals whose behavior is unfavorable or incorrect ("bad") for the observer. Such distinguished "bad" individuals should be adequately treated (e.g. convicted, avoided, liquidated) which should also be formed by a division profile. In the case of a multiagent system, it is possible to equip every agent in the system with division profile mechanisms, so the security is assured by all agents existed in the system. Division profile is defined as:

$$
a_{d}=\left(M_{d}, Q_{d}, S_{d}\right)
$$

where $M_{d}$ is a set of division states $m_{d}$ of agent $a, Q_{d}$ is a configuration of goals $q_{d}$ of agent's a division profile, $S_{d}$ is a configuration of strategies $s_{d}$ of agent's $a$ division profile.

\subsection{Division State}

Division state $m_{d}$ of agent $a$ is represented as a vector:

$$
m_{d}=\left(m_{d}^{1}, m_{d}^{2}, \ldots, m_{d}^{j-1}, m_{d}^{j}\right)
$$

where $j$ is the number of neighboring agents; neighboring agents are agents which are visible for agent $a$ (including itself, if all agents in the system are visible for agent $a, j$ is equal to the number of all existing agents in the system); $m_{d}^{k}$ is the factor subordinated to neighboring agent number $k$; this factor can be a number from any range and it indicates whether the agent number $k$ is "good" or "bad" (low number indicates "good", high number indicates "bad").

\subsection{How to Fix the Division State}

To fix division state (or to distinguish between "bad" and "good" individuals) some mechanisms of the immune system can be used. Fixing of division state is 
inspired by immunological mechanisms - generation of $\mathrm{T}$ cells in the immune system. This article does not present an artificial immune system, but some immunological mechanisms are used in a part of presented solutions. Immunological mechanisms should operate on actions made by observed agents. This approach is opposite to the one proposed in e.g. [3,12] in which immunological mechanisms operate on the resource's structure.

Immunological intruders detection in the computer environment has to be done on the basis of certain characteristic structures. These structures in the case of behavior observation can be chains of actions performed by an observed agent. These chains are of the settled length $l$, so one chain contains $l$ objects, which present undertaken actions by observed agent (one object represents one action). We should define the way how agent $a$ will recognize (notice, but not estimate) actions undertaken by neighbors. It is possible to store all actions undertaken by agents in the environment of agent computer system. The action stored should be accompanied by the notion by whom a particular action has been undertaken. This method presumes the mediation of the environment and / or resources of the environment in the process of recognizing undertaken actions.

Detectors. The method of fixing the division state refers to the mechanism of immune system. Once detector's set is generated, this detector's set is used to find "bad" among presented sequences of action-objects.

In order to generate a set of detectors $R$, own collection $W$ should be specified. This collection includes correct, "good" sequences of action-objects. This collection $W$ should consist of action-object sequences of length $l$, which is undertaken by the agent-observer. This is correct, because of the assumption that actions which the agent undertakes are evaluated as "good" by him. Presuming there are stored $h$ last actions undertaken by every agent, own collection $W$ will contain $h-l+1$ elements.

Algorithm of Detectors Generation. The algorithm of detectors generation refers to the negative selection - the method in which $\mathrm{T}$-lymphocytes are generated. From set $R_{0}$ of generated sequences of length $l$ those reacting with any sequence from collection $W$ are rejected. Set $R_{0}$ contains every possible sequence (but it is also possible to use a set of random generated sequences). Sequence reaction means that elements of those sequences are the same. Sequences from set $R_{0}$ which will pass such a negative selection create a set of detectors $R$.

Behavior Estimation of Neighboring Agents. First stage is a neighbor observation during which actions (and order of those actions) executed by neighboring agents are remembered. Those remembered actions create sequence $N$ of presumed length $h$. After the next stage of detectors generation, generated detectors are used to find "bad", unfavorable agents. Every subsequence $n$ of length $l$ of sequence $N$ is compared with every detector $r$ from set $R$, as it is shown in Fig. 1 If sequence $n$ and $r$ match, it means finding "bad", unfavorable actions. Sequence 


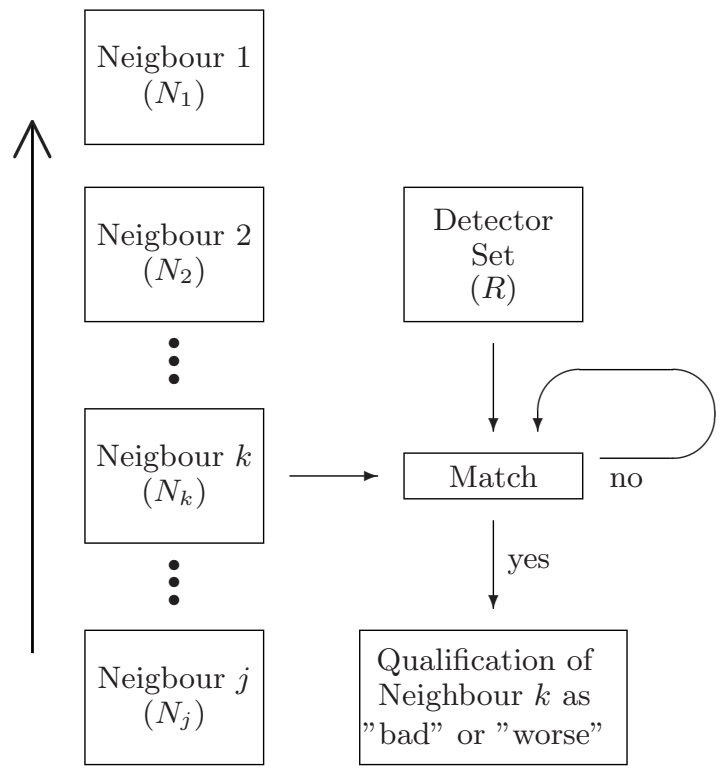

Fig. 1. Process of behavior estimation of neighboring agents, process presented for agent number $k$

matching means that the elements of the sequences compared are the same. The number of matches for every observed agent should be counted. On this basis behavior estimation is made - division state $m_{d}=\left(m_{d}^{1}, m_{d}^{2}, \ldots, m_{d}^{j-1}, m_{d}^{j}\right)$ of agent-observer is modified to the $m_{d}{ }^{\prime}=\left(m_{d}^{1^{\prime}}, m_{d}^{2^{\prime}}, \ldots, m_{d}^{j-1^{\prime}}, m_{d}^{j^{\prime}}\right)$, where $j$ is the number of agents in the environment, $m_{d}^{k^{\prime}}$ is assigned to the number of counted matches for agent number $k$.

\subsection{Configuration of Goals of Agent's Division Profile}

The way neighboring agents are treated is described by $Q_{d}$ - configuration of goals $q_{d}$ of agent's division profile. Configuration of goals of an agent is constant (however it is possible to design such a system, which in is possible the goal's adaptation). In the system described the configuration of goals consists only from one goal - liquidation neighboring agent (or agents) number $k$, if $m_{d}^{k}=$ $\max \left(m_{d}^{1}, m_{d}^{2}, \ldots, m_{d}^{j-1}, m_{d}^{j}\right)$.

\subsection{Configuration of Strategies of Agent's Division Profile}

Actions, which should be undertaken by agent $a$ in order to treat agent number $k$ in the way described by the configuration of goal, are specified by $S_{d}$ - the configuration of strategies $s_{d}$ of agent's division profile. The configuration of 
strategies of the agent is constant and in the system described the configuration of strategies consists only of one goal: if the goal is to liquidate agent number $k$, a demand of deleting agent number $k$ is send to the environment (coefficient $o_{d}$ equal to the $m_{d}^{k}$ is attributed to this demand).

This configuration of strategies presumes an intervention of system's environment in the liquidation of the agent. In the systems described the environment calculates the sum of coefficients for every agent separately attributed to demands and liquidates all agents which have the maximum sum of coefficients and this sum is larger than constant $O U$. Periodically, after a constant time period, the calculated sums of coefficients are set to 0 . Constant coefficient $O U$ is introduced in order to get tolerance for behavior that is evaluated as "bad" in a short time, or is evaluated as "bad" by a small amount of agents.

\section{Experiment}

In the computer system there are some operation which must be executed in couples, for example: open and close a file, connection request and disconnection request. There are a lot of attack techniques which consist in doing only one from a couples (or trios...) of obligatory operations (for example so-called SYN flood attack [11]). There is simulated a system with two types of agents:

- good agents which perform some operations in couples (e.g. open, open, open, close, open, close, close, close);

- bad agents which perform only one from a couples of some operations (e.g. open, open, open, open, open, open, open, open).

In the simulation there is no possibility of distinguishing the type of an agent on the basis of the agent's structure. So the only possibility is to observe the agent's behavior and process the actions observed (actions-objects) to distinguish whether the agent is good or bad.

\subsection{Simulation - Unsecured Computer System}

First a case was simulated, in which only good agents exist in the computer system - initially there are 80 good agents in the system, which do not have any security mechanisms. Next a case was simulated, in which good and bad unsecured agents exist in the computer system — initially there are 64 good agents and 16 bad agents, all agents do not have any security mechanisms. The system in those two cases was simulated to 300 time periods and there were 10 simulations performed. Diagram in Fig. 2 shows the average numbers of agents in separate time periods.

If there are not any bad agents in the simulated system, all good agents can exist without any disturbance. The existence of bad agents in the system causes problems with executing tasks by good agents, which die after some time periods. Bad agents still remain in the system, which is blocked by those bad agents. 


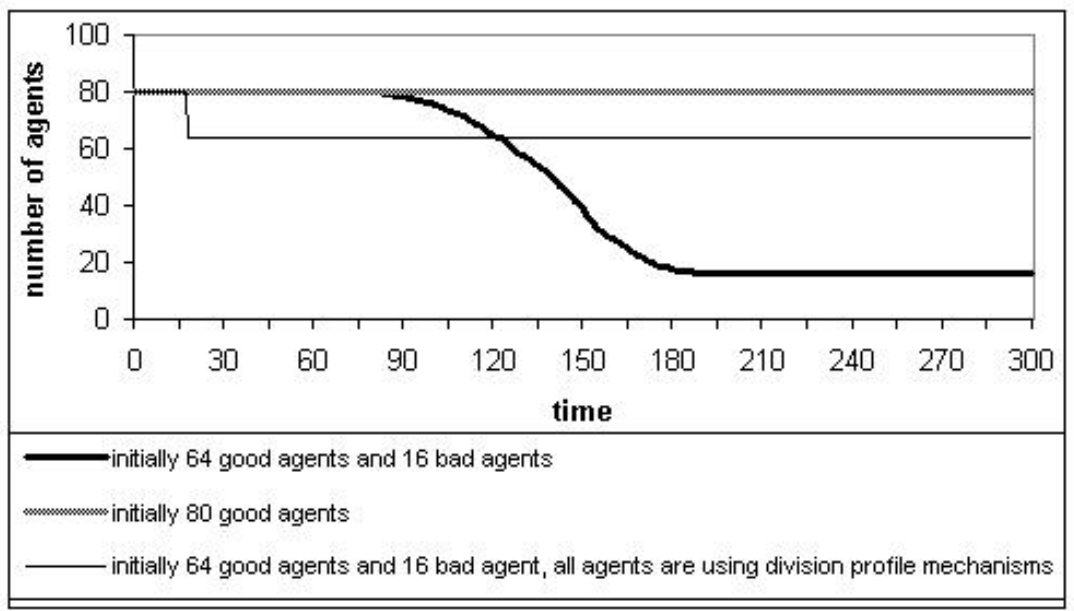

Fig. 2. Number of agents in separate time periods

\subsection{Simulation — Secured Computer System}

A case was simulated, in which good and bad secured agents exist in the computer system - initially there are 64 good agents and 16 bad agents. All agents in the system were equipped with the division profile security mechanisms. The system was simulated to 300 time periods and there were 10 simulations performed. Diagram in Fig. 2] shows the average numbers of agents in separate time periods.

In the environment there are last 18 actions stored undertaken by every agent. After 18 actions have been undertaken by every agent, detectors are constructed of length $l=5$. Agents use their division profile mechanisms to calculate which neighboring agent they want to eliminate. Agent demand to eliminate these neighbors which have the maximum of detector's matchings. Agents present their demands to the environment with the number of matchings. The environment counts matchings in the demands presented and eliminates agents as it was proposed in the description of division profile mechanisms. The constant $O U$ is set up to 480 .

After detectors were constructed, bad agents were distinguished due to the division profile mechanisms. In the same time period, recognized agents were deleted, what makes it possible for agents belonging to good type to function freely.

\section{Conclusion}

This paper presents a discussion of security problem in the computer system. Security solutions which create new security paradigms were proposed: 
- equip all system resources (e. g. agents, programs) with security mechanisms,

- security mechanisms should be based on activity observation rather than looking for some fragments of code (signatures),

- design the environment of computer system in such a way so as to support security mechanisms with which system's resources are equipped.

In this paper security mechanisms with immunological approach were presented which fulfill the said security paradigms. All these security mechanisms were called a division profile. The conception presented was simulated and the obtained results confirm the effectiveness of this solution. The simulation enables to anticipate how the described mechanisms will function in the real world of computer systems.

Security mechanisms designed on the grounds of the conception presented have such advantages as detection of previously unseen danger activities, detection based on activity observation and decentralized detection.

\section{References}

1. Cetnarowicz K., Nawarecki E., Żabińska M.: M-agent Architecture and its Application to the Agent Oriented Technology. Proc. of the DAIMAS'97, St. Petersburg (1997)

2. Cetnarowicz K., Rojek G., Werszowiec-Plazowski J., Suwara M.: Utilization of Ethical and Social Mechanisms in Maintenance of Computer Resources' Security. Proc. of the Agent Day 2002, Belfort (2002)

3. Forrest S., Perelson A. S., Allen L., Cherukuri R.: Self-nonself Discrimination in a Computer. In Proc. of the 1994 IEEE Symposium on Research in Security and Privacy, IEEE Computer Society Press, Los Alamitos (1994) 202-212

4. Forrest S., Perelson A. S., Allen L., Cherukuri R.: A Change-detection Algoritm Inspired by the Immune System. IEEE Transactions on software Engineering, IEEE Computer Society Press, Los Alamitos (1995)

5. Gibbs W. W.: Jak przetrwać w niebezpiecznym świecie? Świat nauki, Wydawnictwo Prószyńska i s-ka (2002)

6. Hofmeyr S. A., Forrest S.: Architecture for an Artificial Immune System. Evolutionary Computation, vol. 7, No. 1 (2002) 45-68

7. Kim J., Bentley P.: The Human Immune System and Network Intrusion Detection. 7th European Congress on Intelligent Techniques and Soft Computing (EUFIT '99) Aachen September 13-19 (1999)

8. Kim J., Bentley P.: Negative Selection within an Artificial Immune System for Network Intrusion Detection. The 14th Annial Fall symposium of the Korean Information Processing Society, Seoul October 13-14 (2000)

9. Ossowska M.: Normy moralne. Wydawnictwo Naukowe PWN, Warszawa (2000)

10. Ricken F.: Etyka Ogólna. Wydawnictwo ANTYK - Marek Derewiecki, Kety (2001)

11. Schetina E., Green K., Carlson J.: Bezpieczeństwo w sieci. Wydawnictwo HELION, Gliwice (2002)

12. Wierzchoń S. T.: Sztuczne systemy immunologiczne: teoria i zastosowania. Akademicka Oficyna Wydawnicza Exit, Warszawa (2001) 